\title{
Proarrhythmia in KCNJ2 E299V-linked Short QT Syndrome: A Simulation Study
}

\author{
Cunjin Luo ${ }^{1,2}$, Tong $\mathrm{Liu}^{3}$, Ying $\mathrm{He}^{4}$, Kuanquan Wang ${ }^{5}$, Henggui Zhang ${ }^{6}$ \\ ${ }^{1}$ School of Computer Science and Electronic Engineering, University of Essex, Colchester, UK \\ ${ }^{2}$ Key Lab of Medical Electrophysiology, Ministry of Education, Institute of Cardiovascular Research, \\ Southwest Medical University, Luzhou, China \\ ${ }^{3}$ Second Hospital, Tianjin Medical University, Tianjin, China \\ ${ }^{4}$ School of Computer Science, University of Nottingham, Nottingham, UK \\ ${ }^{5}$ School of Computer Science and Technology, Harbin Institute of Technology, Harbin, China \\ ${ }^{6}$ School of Physics and Astronomy, University of Manchester, Manchester, UK
}

\begin{abstract}
Short QT syndrome (SQTS) is a clinical disorder associated with cardiac arrhythmias and sudden cardiac death (SCD). Short QT syndrome variant 3 (SQT3) has been linked to the D172N or E299V gain-in-function mutation to Kir2.1, which preferentially increases outward current through channels responsible for inward rectifier $K^{+}$current $\left(I_{K 1}\right)$. There is a novel blocker of Kir2.1, Styrax, which is a kind of natural compound selected from traditional Chinese medicine. In this study, the ten Tusscher et al model of ventricular action potential was used to investigate the potential effects of Styrax on the short QT syndrome associated with the Kir2.1 D172N mutation and E299V mutation. Our data showed that Styrax can prolong the action potential $(A P)$ and $Q T$ interval on the ECG under the condition of SQT3 associated with D172N and E299V mutations. We suggested that Styrax may be a potential drug for the treatment of SQT3.
\end{abstract}

\section{Introduction}

Short QT syndrome (SQTS) is a rare channelopathy, characterized by a short QT interval on the ECG, and patients with this disorder are predisposed to a higher risk of develop cardiac arrhythmias and even sudden cardiac death (SCD) [1-3]. Although significant progress has been made in recent years to better understand the mechanism of this disorder, there are still significant challenges for the treatment of SQTS [4].

SQTS is genetically heterogeneous, with a genotypephenotype relationship. Since the first description in 2000 [4], associated mutations have been identified in eight different genes: SQT1 variant to SQT3 variant are associated with gain-of-function mutations in potassium channels, SQT4 variant to SQT6 variant are associated with loss-of-function of calcium channels, SQT7 variant is associated with a loss-of-function in SCN5A, SQT8 variant is associated with a mutation in the cardiac CL/HCO3 exchanger AE3.

SQT1 variant was caused by a gene mutation (N588K) to the hERG encoding the rapid delayed rectifier potassium $\left(I_{\mathrm{Kr}}\right)$, SQT2 variant was caused by a gene mutation (V307L) to the KCNQ1 encoding the slow delayed rectifier potassium $\left(I_{\mathrm{Ks}}\right)$, and SQT3 variant was caused by a gene mutation (D172N, E299V) to the KCNJ2 encoding the inward rectifier potassium current $\left(I_{\mathrm{K} 1}\right)$. However, effective management of SQTS remains challenging.

The anti-arrhythmic drug quinidine is used as the frontline therapy for SQT1 patients, and literature shows that chloroquine has been suggested as a drug for SQT3 [5]. A novel blocker of Kir2.1, Styrax, was identified as a kind of natural compound selected from traditional Chinese medicine [6]. However, pharmacotherapeutic effects of Styrax on SQT3 variant have not been investigated. The aim of this study was to assess and predict the possible effects of Styrax on human ventricular patho-electrophysiology associated with SQT3 variants linked D172N and E299V mutations.

\section{Methods}

Human ventricular electrophysiology was simulated by using the ten Tusscher et al model [7]. A mathematical formulation for $I_{\mathrm{K} 1}$ in the control action potential was modified to fit current densities obtained from WT Kir2.1 channels, expressed in HEK cells [8, 9].

Using the same approach as detailed for amiodarone, quinidine and chloroquine in our previous study $[1,10]$, a simple pore block model was developed, incorporating 
known actions of Styrax on $I_{\mathrm{K} 1}$. Styrax is a Kir2.1 channel blocker, and was modelled by using half maximal inhibitory concentration $\left(\mathrm{IC}_{50}\right)$ values from the literature. Styrax $(0.017 \mu \mathrm{M})$ blocked the outward current of the mutants (D172N and E299V) of Kir2.1 $\left(I_{\mathrm{K} 1}\right)$ by $60 \%$ and $42 \%$, respectively. Propagation of excitation waves in cardiac tissue was described using the mono-domain equation,

$$
C_{m} \frac{\partial V}{\partial t}=-\left(I_{i o n}+I_{s t i m}\right)+\nabla \cdot(D \nabla V)
$$

The tissue model comprised an ENDO ventricular region, a MIDDLE ventricular region, and an EPI ventricular region. The tissue was $15 \mathrm{~mm}$ long with 100 nodes that were spaced $0.15 \mathrm{~mm}$ apart, with each node representing a $150-\mu \mathrm{m}$ cylindrical cell. Equation was solved using finite difference PDE solver based on the explicit forward Euler method. Electrical excitation waves were produced by using tissue model. The ECG was calculated by the method proposed by Gima and Rudy [11].

\section{Results}
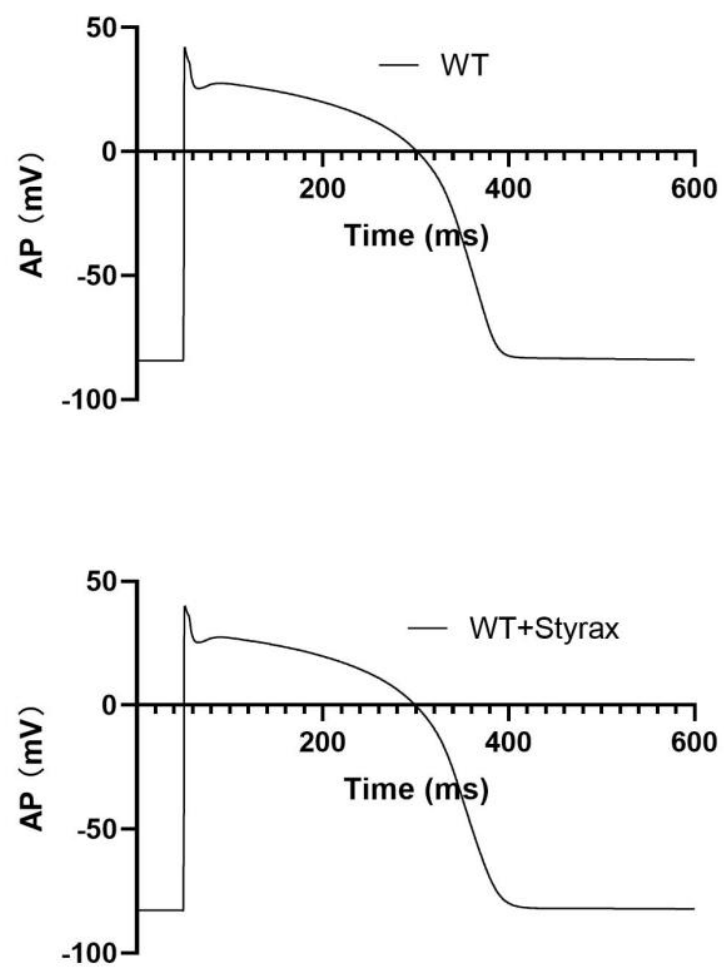

\subsection{Single cell simulations}

The human ventricular action potential (AP) duration at a pacing rate of $1.25 \mathrm{~Hz}$ frequency under SQT3 D172N and E299V mutations, and Styrax-in-action conditions was shown in Figure 1 and Figure 2, respectively. The plots show the action potential $\mathrm{V}(\mathrm{mV})$ versus time $\mathrm{t}(\mathrm{ms})$. Changes in $I_{\mathrm{K} 1}$ due to the Kir2.1 D172N or E299V mutations abbreviated human ventricular APD. Styrax prolonged the human ventricular APD under SQT3 D172N and E299V mutation conditions.

\subsection{Tissue simulations}

In tissue simulations, we simulated the ECGs under SQT3 D172N and E299V mutations, and Styrax-in-action conditions at a stimulation frequency of $1.25 \mathrm{~Hz}$, shown in Figure 3 and Figure 4, respectively. It substantiated a causal link between the KCNJ2-D172N or E299V mutations and QT interval shortening. Styrax prolonged the QT intervals on the ECG under SQT3 D172N and E299V mutation conditions.
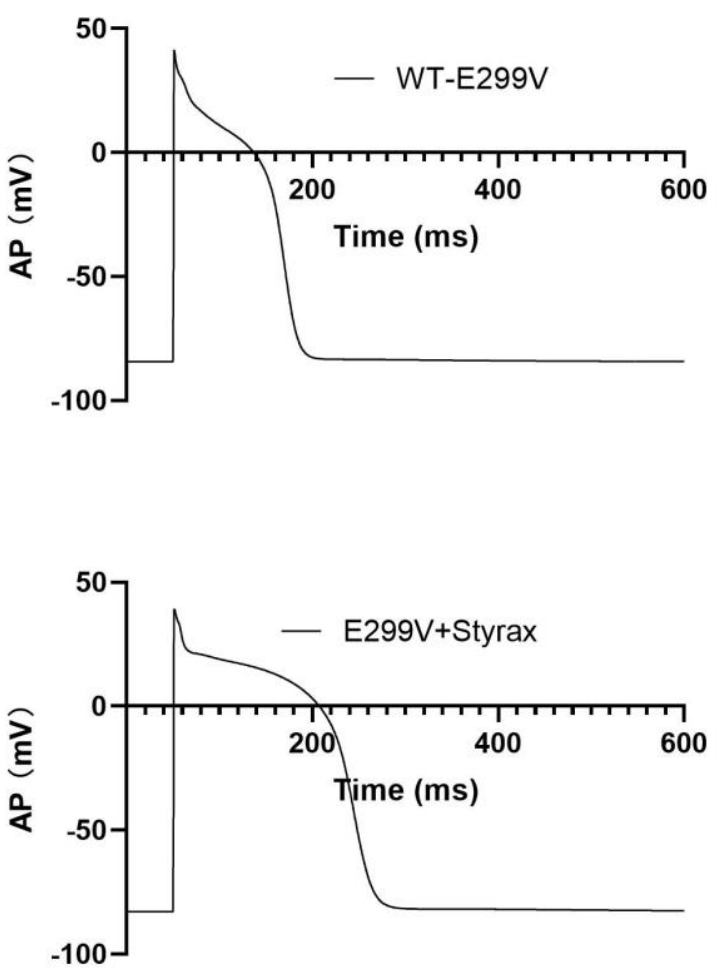

Figure 1. Action potentials under the E299V mutation with the action of Styrax 

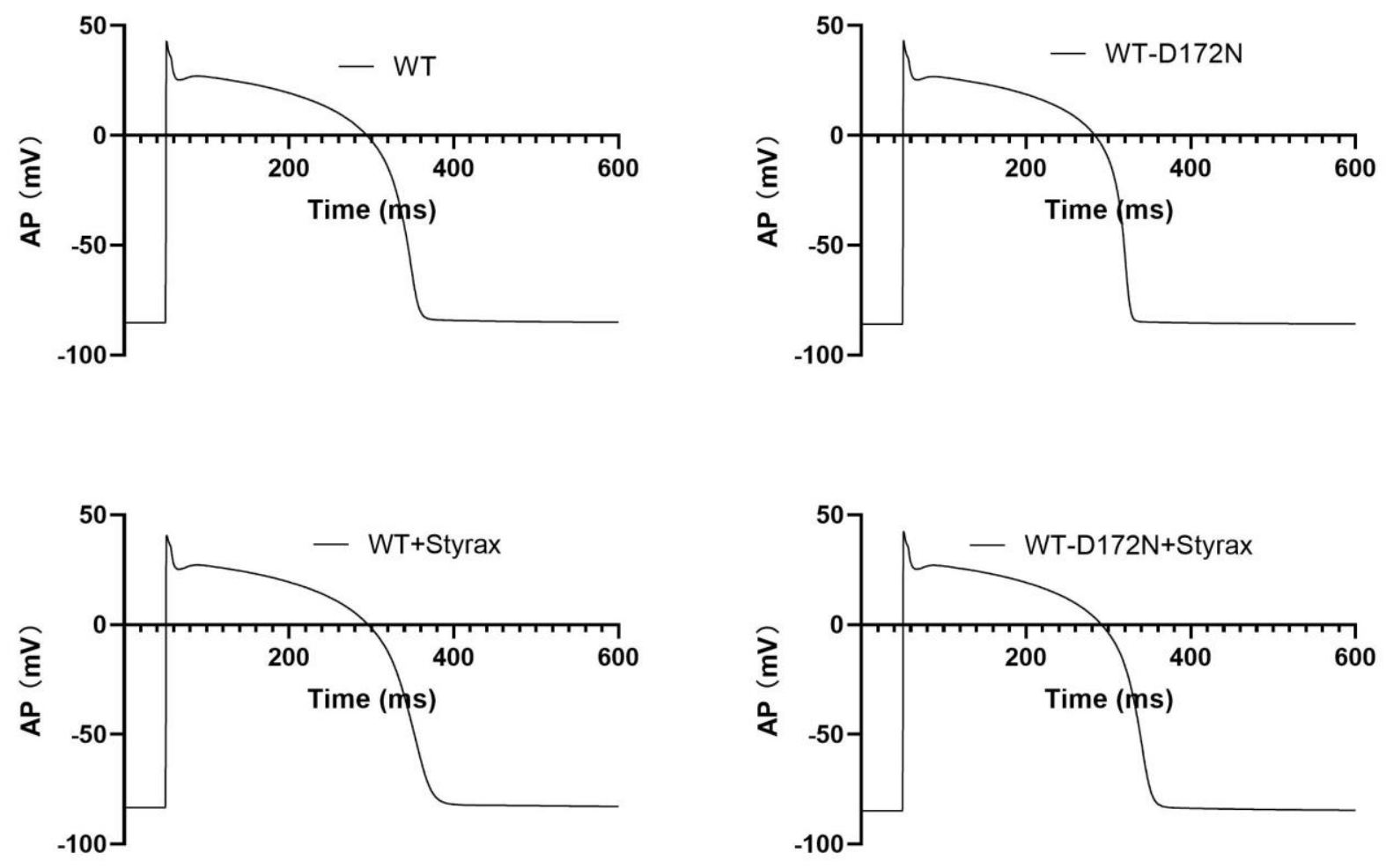

Figure 2. Action potentials under the D172N mutation with the action of Styrax.
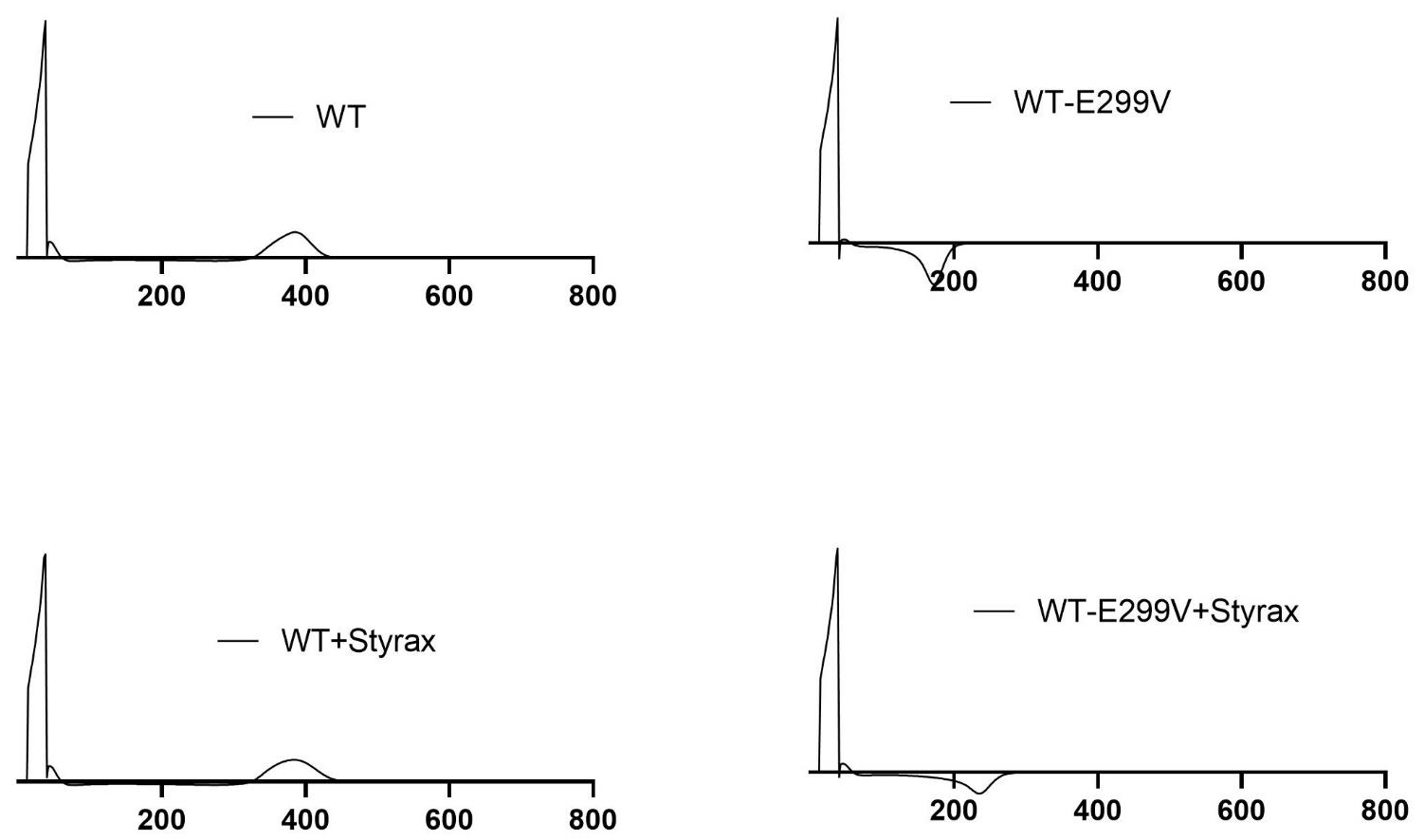

Figure 3 ECGs under the E299V mutation with the action of Styrax 

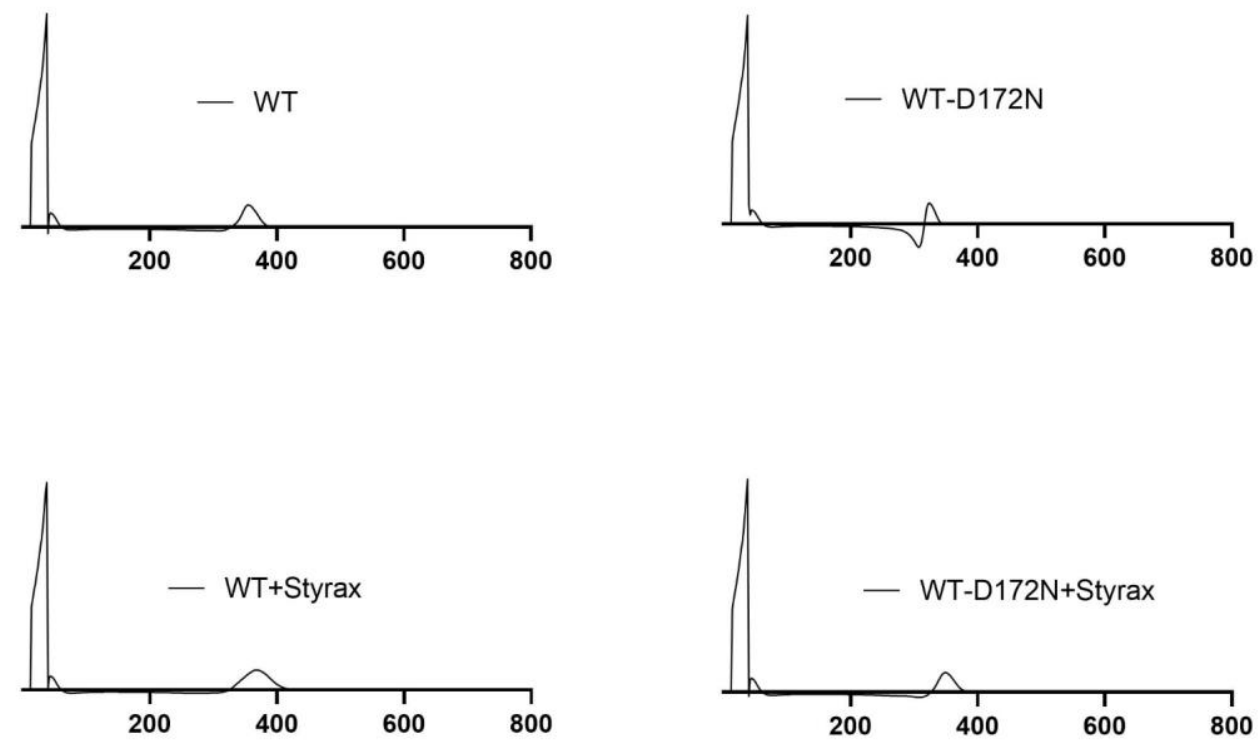

Figure 4 ECGs under the E299V mutation with the action of Styrax

\section{Conclusion and Future Work}

In this study, the actions of the drug Styrax was simulated by using a computational approach, successfully demonstrated how the virtual heart model as a platform for the drug screening. From our simulation results, we conclude that Styrax may be an effectively drug on the short QT syndrome variant 3 associated with D172N and E299V mutations. In future work, we will consider simulating different doses of Styrax to assess the potential effects on SQT3, and also consider simulating the effects of Styrax on the reentrant waves under SQT3.

\section{Acknowledgments}

This work was supported by the National Natural Science Foundation of China (No. 61803318, 81970270), and Scientific-Technological Collaboration Project under Grant No. 2018LZXNYD-FP02.

\section{References}

[1] C Luo, K Wang, H Zhang. Modelling the effects of quinidine, disopyramide, and E-4031 on short QT syndrome variant 3 in the human ventricles. Physiological measurement 38 (10), 1859.

[2] K Wang, C Luo, Y Yuan, W Lu, H Zhang. Simulation of reentrant wave dynamics in a 2-D sheet of human ventricle with KCNJ2-linked variant 3 short QT syndrome. Computing in Cardiology 2014, 61-64.

[3] C Luo, K Wang, Y Liu, Y Xia, H Zhang. Effects of quinidine on short QT syndrome variant 2 in the human ventricle: a modelling and simulation study. 2017 Computing in
Cardiology (CinC), 1-4.

[4] I El-Battrawy, J Besler, X Li, et al. Impact of Antiarrhythmic Drugs on the Outcome of Short QT Syndrome. Frontiers in pharmacology, 10 (2019), 771.

[5] C Luo, K Wang and $\mathrm{H}$ Zhang. Modelling the effects of chloroquine on KCNJ2-linked short QT syndrome. Oncotarget, 8, 63 (Dec 5 2017), 106511-106526.

[6] S Ren, C Pang, J Li, et al. Styrax blocks inward and outward current of Kir2.1 channel. Channels (Austin). 2017; 11(1): 46-54.

[7] ten Tusscher, K. H. and Panfilov, A. V. Alternans and spiral breakup in a human ventricular tissue model. Am J Physiol Heart Circ Physiol, 291, 3 (Sep 2006), H1088-1100.

[8] I Adeniran, A El Harchi, J Hancox, H Zhang. Proarrhythmia in KCNJ2-linked short QT syndrome: insights from modelling. Cardiovascular Research, Volume 94, Issue 1, 1 April 2012, Pages 66-76.

[9] M Deo, Y Ruan, S Pandit, et al. KCNJ2 mutation in short QT syndrome 3 results in atrial fibrillation and ventricular proarrhythmia. PNAS March 12, 2013110 (11) 4291-4296.

[10] C Luo, K Wang, M Yuan, et al. Effects of amiodarone on ventricular excitation associated with the KCNJ2-linked short QT syndrome: Insights from a modelling study. 2015 Computing in Cardiology Conference (CinC), 1093-1096.

[11] K Gima, Y Rudy. Ionic current basis of electrocardiographic waveforms: a model study. Circulation research, 90, 8 (May 3 2002), 889-896.

Correspondence:

Cunjin Luo

Email: cunjin.luo@essex.ac.uk

School of Computer Science and Electronic Engineering

University of Essex

Colchester CO4 3SQ

UK 